\title{
AVALIAÇÃO TOMOGRÁFICA DO POSICIONAMENTO DE PARAFUSOS \\ PEDICULARES EM DEFORMIDADES NA COLUNA TORÁCICA E LOMBAR INTRODUZIDOS COM BASE NA TÉCNICA "FREE HAND"
}

\author{
TOMOGRAPHIC EVALUATION OF THE PLACEMENT OF PEDICLE SCREWS USED IN DEFOR- \\ MITIES OF THE THORACIC AND LUMBAR SPINE BASED ON THE "FREE HAND" TECHNIQUE \\ EVALUACIÓN TOMOGRÁFICA DE LA COLOCACIÓN DE TORNILLOS PEDICULARES EN DE- \\ FORMIDADES DE LA COLUMNA TORÁCICA Y LUMBAR, INTRODUCIDOS CON BASE EN LA \\ TÉCNICA "FREE HAND"
}

Dante Bernardes Giubileil ${ }^{1}$, Paulo Tadeu Maia Cavali², Maurício Antonelli Lehoczkl ${ }^{3}$, Alexander Junqueira Rossato ${ }^{3}$, Marcelo ltalo Risso-Neto ${ }^{4}$, Guilherme Rebechi Zuiani ${ }^{4}$ Ivan Guidolin Veiga ${ }^{4}$, Wagner Pasoualin ${ }^{4}$, Élcio Landim ${ }^{5}$

\begin{abstract}
RESUMO
Objetivo: Avaliar o posicionamento de parafusos pediculares aplicados em vértebras da coluna torácica e lombar em pacientes portadores de escolioses, introduzidos pela técnica "free hand". Métodos: Avaliação de quinze tomografias computadorizadas de 284 parafusos pediculares em 15 pacientes (sete homens e oito mulheres), com idade entre 12 e 39 anos (média de 16,7 anos) com escoliose. Os parafusos foram inseridos de T2 a S1 para as seguintes afecções: duas escolioses congênitas e 13 escolioses neuromusculares, visando avaliar o posicionamento dos parafusos com relação às paredes dos pedículos. Resultados: Em relação às corticais do pedículo, 244 parafusos (86\%) foram posicionados entre as corticais ou apenas as tocaram. Houve lesão da cortical lateral em 16 parafusos e lesão da cortical medial em 13 parafusos, sendo maior o número de violações nas vértebras torácicas. Conclusão: $\mathrm{O}$ uso de parafusos pediculares na instrumentação de vértebras torácicas e lombares nas escolioses mostrou -se segura e efetiva quando aplicado em diferentes níveis com a técnica "free hand". Nas vértebras torácicas as violações da cortical pedicular foram mais frequentes, mas não prejudicaram a estabilidade da fixação e não lesaram estruturas neurais, vasculares e viscerais.
\end{abstract}

Descritores: Vértebras torácicas; Vértebras lombares; Escoliose; Parafusos ósseos.

\section{ABSTRACT}

Objective: To evaluate the placement of pedicle screws in the vertebrae of the thoracic and lumbar spine in patients with scoliosis using the "free hand" technique. Methods: Evaluation of CT scans of 284 pedicle screws in 15 patients (seven men and eight women) aged between 12 and 39 years (mean 16.7 years) with scoliosis. The screws were inserted from T2 to S1 for the following conditions: two congenital scoliosis and 13 neuromuscular, to evaluate the placement of screws in relation to the walls of pedicles. Results: Regarding the cortical pedicle screws, 244 (86\%) were positioned between the cortical or just touching it. Lateral cortical lesion was seen in 16 screws and medial cortex lesion in 13 screws, being greater the number of violations in the thoracic vertebrae. Conclusion: The use of pedicle screw instrumentation in thoracic and lumbar vertebrae for scoliosis proved safe and effective when applied to different levels with the "free hand" technique. In the thoracic vertebrae, the pedicular cortical violations were more frequent, but did not impair the stability of fixation and did not injure the neural, vascular and visceral structures.

Keywords: Thoracic vertebrae; Lumbar vertebrae; Scoliosis; Bone screws.

RESUMEN

Objetivo: Evaluar la colocación de tornillos pediculares utilizados en las vértebras de la columna torácica y lumbar en pacientes con escoliosis, introducidos mediante la técnica "free hand". Métodos: Se evaluaron quince tomografías computadorizadas de 284 tornillos pediculares en 15 pacientes (siete hombres y ocho mujeres), de edades comprendidas entre 12 y 39 años (promedio de 16,7 años), que tenían escoliosis. Los tornillos se insertaron desde T2 a S1, siendo dos escoliosis congénitas y 13 escoliosis neuromusculares; se analizó la colocación de los tornillos en relación a las paredes de los pedículos. Resultados: Cuanto a las corticales del pedículo, 244 (86\%) de los parafusos fueron colocados entre las corticales o apenas las tocaron. Lesión cortical lateral se observó en 16 tornillos y lesión cortical medial en 13 tornillos. Fue mayor el número de violaciones torácicas. Conclusión: El uso de tornillos pediculares en la instrumentación de las vértebras torácicas y lumbares en la escoliosis demostró ser seguro y eficaz cuando se aplican a diferentes niveles con la técnica de "free hand". En las vértebras torácicas, las violaciones de la cortical pedicular fueron más frecuentes, pero sin poner en peligro la estabilidad de la fijación y sin dañar estructuras neurales, vasculares y viscerales.

Descriptores: Vértebras torácicas; Vértebras lumbares; Escoliosis; Tornillos óseos.

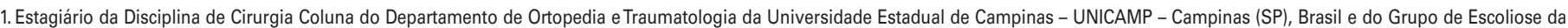
Associação de Assistência à Criança Deficiente - AACD - São Paulo (SP), Brasil.

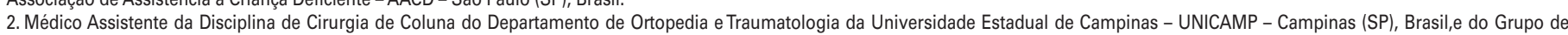
Escoliose da Associação de Assistência à Criança Deficiente - AACD.

3. Médico Assistente do Grupo de Escoliose da Associação de Assistência à Criança Deficiente - AACD - São Paulo (SP), Brasil.

4. Médico Assistente da Disciplina de Cirurgia de Coluna do Departamento de Ortopedia eTraumatologia da Universidade Estadual de Campinas - UNICAMP - Campinas (SP), Brasil.

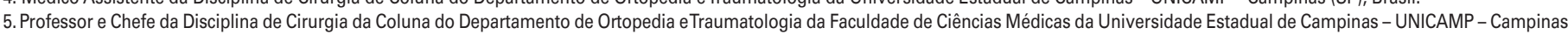
(SP), Brasil, e Chefe do Grupo de Escoliose da Associação de Assistência à Criança Deficiente - AACD - São Paulo (SP), Brasil. 


\section{INTRODUÇÃO}

Nas ultimas duas décadas a instrumentação da coluna vertebral evoluiu com uso de parafusos pediculares ${ }^{1}$. Sua utilização iniciou na coluna lombar, assim os cirurgiões tornaram-se cada vez mais confiantes, despertando interesse nos estudos da morfometria pedicular, incluindo o uso de parafusos pediculares na coluna torácica. O que ampliou o seu uso para variadas patologias da coluna vertebral, como traumáticas, oncológicas, degenerativas, bem como na correção de deformidades ${ }^{2-4}$.

A imprecisão na inserção de parafusos pediculares, além de reduzir a estabilidade do sistema, pode ocasionar dano neurológico, vascular e visceral ${ }^{5}$. Assim, o cirurgião procura utilizar recursos que permitam identificar adequadamente o seu ideal posicionamento, o que inclui referenciais anatômicos, estudos de radiologia simples, radioscopia, tomografia computadorizada e navegação estereotáxica ${ }^{1,6}$.

Desta forma, torna-se cada vez mais importante a busca de técnicas cirúrgicas que sejam seguras, de simples realização, com baixo custo e principalmente, que sejam usadas com confiabilidade pelos cirurgiões ${ }^{3}$

A técnica "free-hand" foi descrita por Kim et al. ${ }^{2}$, na qual a colocação dos parafusos nos pedículos é baseada em parâmetros anatômicos e na sensibilidade tátil do cirurgião para encontrar o centro dos pedículos. Kim encontrou com esta técnica baixas complicações, porém necessita de uma longa sua curva de aprendizado especialmente quando se trata de deformidades.

O objetivo do presente estudo é avaliar o posicionamento de parafusos pediculares aplicados nas vértebras da coluna torácica e lombar em pacientes portadores de escolioses, introduzidos pela técnica "free hand".

\section{MÉTODOS}

Realizado estudo retrospectivo avaliando tomografias computadorizadas realizadas em pacientes submetidos à fixação pedicular.

Foram avaliados 15 pacientes consecutivos operados por escoliose, entre o período de primeiro de novembro de 2010 a 31 de janeiro de 2011. Destes pacientes sete são homens e oito mulheres, com idade entre 12 e 39 anos (média de 16,7 anos).

Os parafusos foram inseridos nos pediculos de T2 ao osso ilíaco em pacientes portadores de escolioses, sendo: duas deformidades congênitas e 13 neuromusculares. A média das curvas escolióticas foi de 74,8 graus de Cobb no pré-operatório.

Todos os exames de tomografia computadorizada selecionados seguiram a técnica definida como rotina pelo Departamento de Radiologia da AACD, a qual realiza corte de dois milímetros paralelos ao eixo dos pedículos. Casos com documentação inadequada foram excluídos do estudo.

Todos os pacientes foram operados, seguindo a técnica preconizada pelo Grupo de Escoliose da AACD, por cirurgiões em treinamento sob supervisão e por cirurgiões com experiência de mais de cinco anos na aplicação de parafusos pediculares utilizando a técnica "free hand".

\section{TÉCNICA}

A colocação dos parafusos é feita com a exposição adequada dos elementos vertebrais posteriores visualizando as facetas articulares e processos transversos.

Nas vértebras torácicas faz-se a remoção da faceta inferior com uso de osteótomo e cureta ${ }^{2}$. Indentifica-se a borda lateral da faceta articular torácica com um marcador. O ponto de entrada do parafuso é a intersecção do terço lateral e mediano da faceta superior da vértebra a ser instrumentada com uma linha média no meio do processo transverso.

Neste ponto insere-se um guia para iniciar a preparação do pedículo, em seguida, avalia-se o pedículo com um palpador, sentindo suas paredes ósseas anterior, lateral, superior e especialmente medial e inferior, pela proximidade com o canal medular e com a raiz nervosa, respectivamente. Após a palpação, insere-se o parafuso pedicular 2,7.

Nas vértebras lombares realiza-se osteotomia do terço látero-inferior da faceta inferior da vértebra superior e o ponto de entrada é o cruzamento da linha que tangência lateralmente a faceta articular superior da vértebra inferior e a linha média do processo transverso $2,7,8$.

Os ângulos de convergência e inclinação crânio-caudal são definidos de acordo com o conhecimento anatômico de cada vértebra e das particularidades relacionadas com o posicionamento do paciente e suas características anatômicas das escolioses ${ }^{2,8}$.

Depois de aplicar os parafusos, avalia-se as posições de inserção, na incidência ântero-posterior e no perfil, através do controle com radioscopia. O parafuso que apresenta sua extremidade cruzando a linha média é investigado, para descartar a possível invasão da região medial do pedículo. No perfil, o parafuso deve estar alinhado com o platô vertebral e não ultrapassar a região anterior do corpo vertebral $\left.\right|^{2,8}$.

No pós-operatório, realiza-se tomografia computadorizada, para avaliar o posicionamento dos parafusos, observando a relação destes com pedículo vertebral.

A análise do posicionamento dos parafusos é realizada por três ortopedistas cirurgiões de coluna e um radiologista, que avaliam as tomografias pós-operatórias independentemente e classificam quanto ao posicionamento de cada parafuso como: sem ou com violação do pedículo, tomando como referência a integridade das corticais pediculares ${ }^{7}$.

As violações do pedículo são qualificadas quanto à posição como superior, inferior, lateral, medial e anterior e quanto à extensão em até dois milímetros, de 2 a 4 milímetros e acima de 4 milímetros.

Nos casos em que houve divergência, as imagens são analisadas novamente e obtido consenso entre os observadores.

\section{RESULTADOS}

Foram avaliados 284 parafusos. Os resultados do posicionamento dos parafusos pediculares estão expostos na Tabela 1. Em relação às corticais do pedículo, 244 parafusos (86\%) foram posicionados entre as corticais ou apenas as tocaram (Figura 1).

Apresentaram lesão da cortical lateral em 16 parafusos (Figura 2) e lesão da cortical medial (Figura 3) em 13 parafusos.

Nove parafusos violaram a cortical entre 0 a $2 \mathrm{~mm}(3,2 \%)$. Vinte e oito (10\%) estavam a 2 a $4 \mathrm{~mm}$; e três $(1,0 \%)$ parafusos estavam acima de $4 \mathrm{~mm}$ (Figura 4 )

Assim 36 (90\%) dos parafusos que violaram a cortical são torácicos e quatro lombares.

Os níveis com maior número de violações foram T6 com sete violações, seguido por T7 com seis violações. Quanto às violações: $13(4,6 \%)$ mediais, 16 (5,6\%) laterais, oito (2,8\%) anteriores (Figura 5).

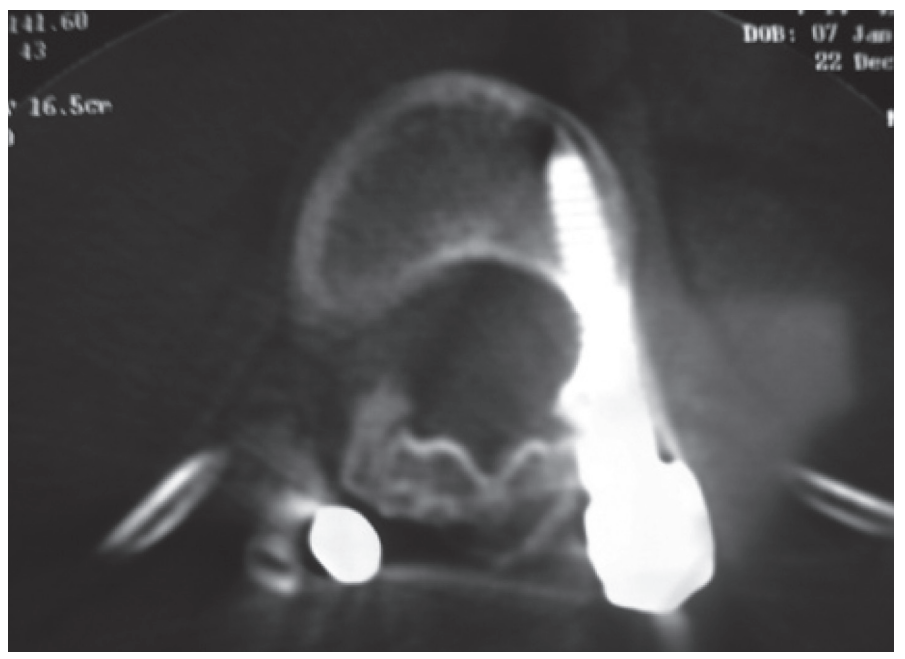

Figura 1. Parafuso pedicular posicionado entre as corticais. 
Tabela 1. Posicionamento dos parafusos pediculares avaliados pela tomografia computadorizada.

\begin{tabular}{c|c|c|c|c|c}
\hline Nível & $\begin{array}{c}\text { Número de } \\
\text { parafusos }\end{array}$ & $\begin{array}{c}\text { Violados } \\
\text { medial }\end{array}$ & $\begin{array}{c}\text { Violados } \\
\text { lateral }\end{array}$ & $\begin{array}{c}\text { Violados } \\
\text { anterior }\end{array}$ & $\begin{array}{c}\text { Violados supe- } \\
\text { rior e inferior }\end{array}$ \\
\hline T2 & 06 & & 2 & & \\
\hline T3 & 13 & & 1 & 1 & \\
\hline T4 & 21 & 3 & 2 & 1 & \\
\hline T5 & 19 & 2 & 2 & 1 & \\
\hline T6 & 21 & 4 & 2 & 1 & \\
\hline T7 & 20 & 1 & 3 & 2 & \\
\hline T8 & 16 & & 2 & 1 & \\
\hline T9 & 15 & & 1 & & \\
\hline T10 & 15 & & & & \\
\hline T11 & 17 & 2 & & & \\
\hline T12 & 16 & & & 1 & \\
\hline L1 & 22 & & & & \\
\hline L2 & 21 & & & & \\
\hline L3 & 22 & 1 & & & \\
\hline L4 & 24 & 2 & 1 & & \\
\hline L5 & 06 & & & & \\
\hline Iliaco & 10 & & & & \\
\hline Total & 284 & 13 & 16 & 8 & \\
\hline & & & & & \\
\hline
\end{tabular}

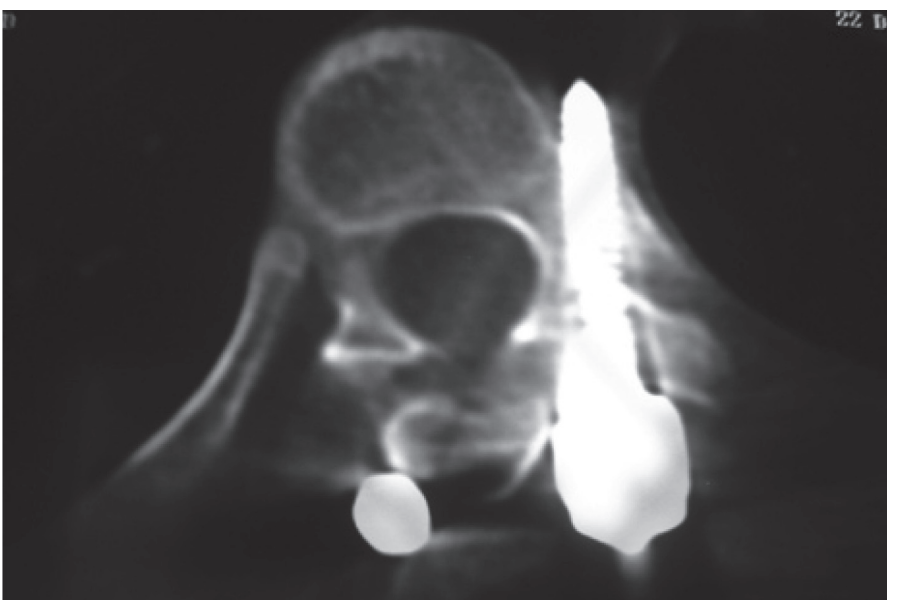

Figura 2. Parafuso pedicular violando a cortical lateral.

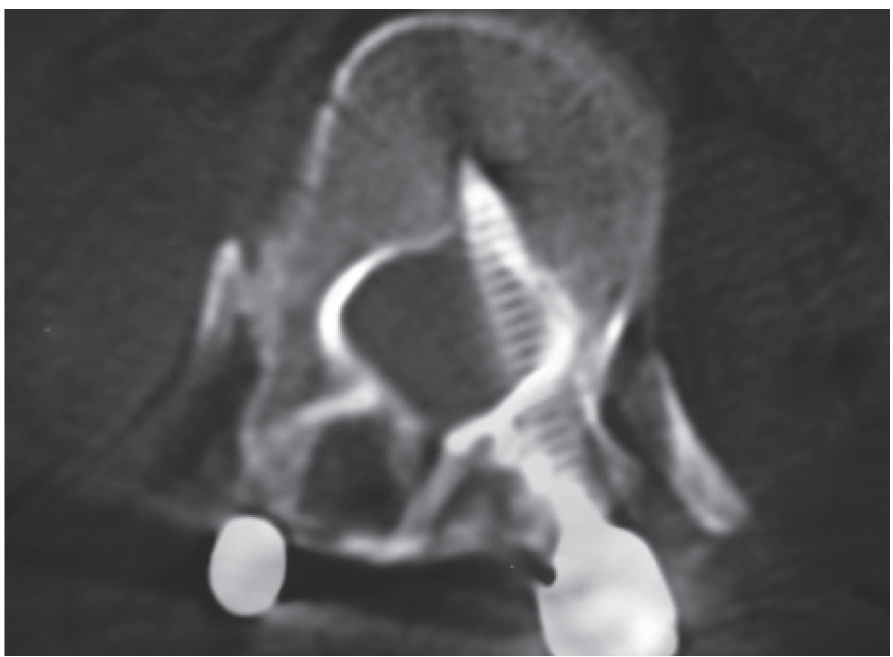

Figura 3. Parafuso pedicular violando a cortical medial.

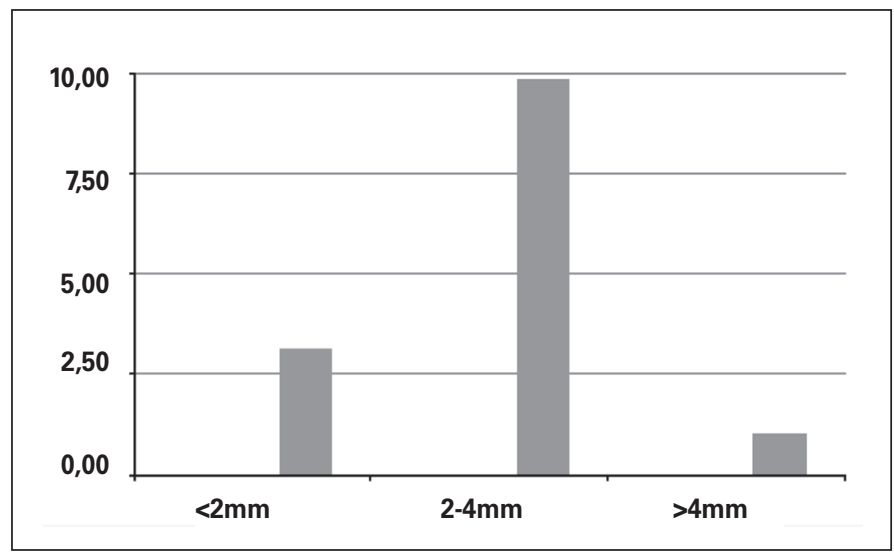

Figura 4. Porcentagem de parafusos violados em relação à parede dos pedículos.

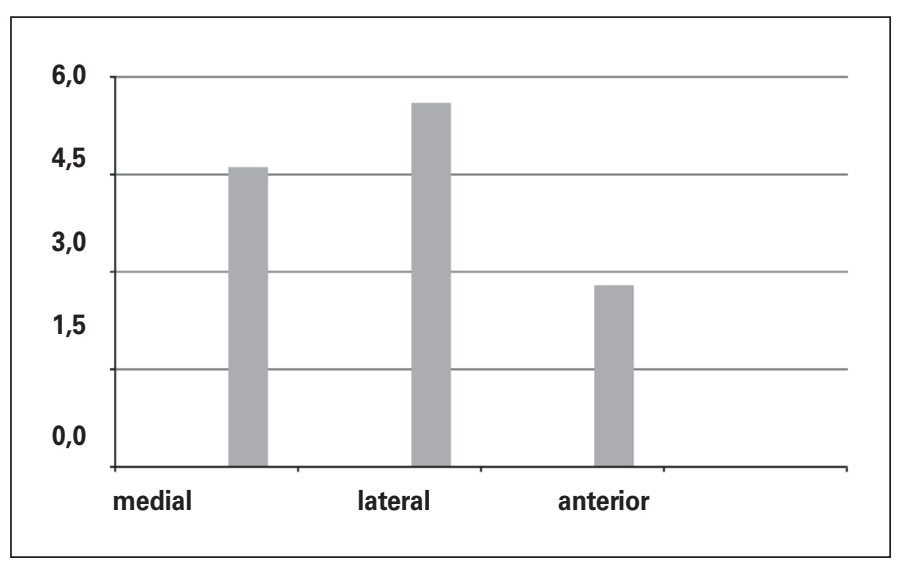

Figura 5. Porcentagens de parafusos que violaram as corticais, laterais e anteriores.

\section{DISCUSSÃO}

A utilização de parafusos pediculares na instrumentação da coluna toracolombar vem se tornando cada vez maior.

A fim de melhorar a acurácia no posicionamento do implante nos pedículos, foram desenvolvidas técnicas de visualização radioscópica biplanar durante a perfuração do trajeto e técnicas de navegação estereotáxica ${ }^{1,4}$. Entretanto, são técnicas que aumentam o tempo de cirurgia, o custo, e expõe o paciente e a equipe cirúrgica a maior radiação $0^{1,4,5,9}$.

$\mathrm{Na}$ literatura a colocação inapropriada de parafusos varia de $3 \%$ a $44 \%$ e foram identificados resultados controversos quanto ao correto posicionamento de parafusos pediculares ${ }^{10}$.

Roy-Camille et al. ${ }^{11}$ reportaram correta localização dos parafusos na coluna lombar em $90 \%$ dos casos, enquanto Castro et al. ${ }^{12}$ apresentaram $60 \%$ de parafusos corretamente localizados.

Kim et al. ${ }^{2}$ encontraram $8 \%$ de mal posicionamento dos parafusos torácicos, sendo 30\% destes parafusos mediais e $70 \%$ laterais.

Em nosso estudo encontramos 14\% de parafusos além das corticais do pedículo, sendo quatro parafusos lombares e 36 parafusos torácicos.

Destes parafusos nenhum causou lesão neurológica, vascular ou visceral, o que concorda com os trabalhos da literatura, que apesar do mal posicionamento dos parafusos, não apresentaram lesão neurológica, vascular ou visceral2, $2,7,13,14$.

Em relação à colocação mais medial do parafuso com lesão da porção medial do pedículo e invasão do canal medular, são descritos na literatura valores entre 1,4\% a 14\% (desde $1 \mathrm{~mm}$ até $8,0 \mathrm{~mm}$ medial) não havendo relato de lesões definitivas, tanto neurológicas,

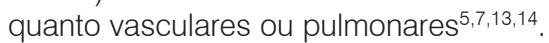


Neste estudo encontramos 5,6\% parafusos mediais, sendo destes parafusos nenhum acima de quatro milímetros.

No estudo de De Marco et al. ${ }^{7}$ foram classificados os parafusos em "aceitos" aqueles que violaram a cortical em até dois milímetros. Outros casos considerados "aceitos" foram aqueles em que os parafusos davam suporte biomecânico à coluna. Em nosso estudo levamos em consideração os critérios de suporte biomecânico somados a ausência de lesões neurológicas, vasculares e viscerais.

As características dos pedículos torácicos nas escolioses podem ter relação com a ausência de complicações mais graves, pois sua parede medial é mais espessa e o ponto de entrada é mais ventral quando comparada à fixação com parafusos lomba- res $^{8}$. A maior dificuldade que encontramos foi a colocação dos parafusos no ápice da curva torácica o que concorda com os dados da literatura ${ }^{5,8}$.

\section{CONCLUSÃO}

O uso de parafusos pediculares na instrumentação de vértebras torácicas e lombares nas escolioses se mostrou segura e efetiva quando aplicado em diferentes níveis com a técnica "free hand". Nas vértebras torácicas as violações da cortical pedicular se mostraram mais frequentes, mas sem prejudicar a estabilidade da fixação e sem expor estruturas neurais, vasculares e viscerais a risco de lesão.

\section{REFERÊNCIAS}

1. Youkilis AS, Quint DJ, McGillicuddy JE, Papadoupoulos SM. Stereotactic navigation for placement of pedicle screws in the thoracic spine. Neurosurgery. 2001; 48(4):771-8.

2. Kim YJ, Lenke LG. Thoracic pedicle screw placement: Free-hand technique. Neurology India. 2005;53(4):512-9.

3. Grauer JN, Vaccaro AR, Brusovanik G, Girardi FP, Silveri CP, Cammisa FP, et al. Evaluation of a novel pedicle probe for the placement of thoracic and lumbosacral pedicle screws. J Spinal Disord Tech. 2004;17(6):492-7.

4. Kim YJ, Lenke LG, Cheh G, Riew KD. Evaluation of pedicle screw placement in the deformed spine using intraoperative plain radiographs: a comparison with computerized tomograpy. Spine (Phila Pa 1976). 2005;30(18):2084-8.

5. Kim YJ, Lenke LG, Bridwell KH, Cho YF, Riew KD. Free hand pedicle screw placement in thoracic spine: is it safe? Spine (Phila Pa 1976). 2004;29(3):333-42.

6. Defino HLA, Mauad Filho J. Estudo morfométrico do pedículo das vértebras torácicas e lombares. Rev Bras Ortop. 1999;34(2):97-108.

7. De Marco FA, Risso Neto MI, Cavali PTM, Sussi MA, Pasqualini W, Landim E, et al. Avaliação do posicionamento de parafusos pediculares na coluna torácica e lombar introduzidos com base em referenciais anatômicos e radioscópicos. Coluna/Columna. 2008;7(1):1-7.

8. Rodrigues LMR, Nicolau JR, Puertas EB, Wajchenberg M. Avaliação tomográ-

fica dos parafusos pediculares torácicos nas escolioses idiopáticas. Coluna/ Columna. 2008;7(2):139-142.

9. UI Haque M, Shufflebarger HL, O’Brien M, Macagno A. Radiation exposure during pedicle screw placement in adolescent idiopathic scoliosis: is fluoroscopy safe? Spine (Phila Pa 1976). 2006;31(21):2516-20.

10. Schwarzenbach O, Berlemann U, Jost B, Visarius H, Arm E, Langlotz F, et al. Accuracy of computer-assisted pedicle screw placement. An in vivo computed tomography analysis. Spine (Phila Pa 1976). 1997;22(4):452-8.

11. Roy-Camille R, Saillant G, Mazel C. Internal fixation of the lumbar spine with pedicle screw plating. Clin Orthop Relat Res. 1986; (203): 7-1.

12. Castro WH, Halm H, Jerosch J, Malms J, Steinbeck J, Blasius S. Accuracy of pedicle screw placement in lumbar vertebrae. Spine (Phila $\mathrm{Pa} 1976)$. 1996; 21(11):1320-4.

13. Hamill CL, Lenke LG, Bridwell KH, Chapman MP, Blanke K, Baldus C. The use of pedicle screw fixation to improve correction in the lumbar spine of patients with idiopathic scoliosis. Is it warranted? Spine (Phila Pa 1976). 1996;21(10):1241-9.

14. Amiot LP, Lang K, Putzier M, Zippel H, Labelle H. Comparative results between conventional and computer-assisted pedicle screw installation in the thoracic, lumbar, and sacral spine. Spine (Phila Pa 1976). 2000;25(5):606-14. 